\title{
Situación energética del cantón Cuenca y líneas de acción preferentes para fortalecer la investigación y el desarrollo a nivel local
}

\author{
John Calle Sigüencia* - Fernando Chica Segovia** \\ email: jcalle@ups.edu.ec
}

\section{Resumen}

El presente artículo se fundamenta en una recopilación de datos a nivel del Ecuador y del Cantón Cuenca en lo que se refiere a generación y uso de la energía eléctrica y al uso de los combustibles fósiles. Todos los datos recopilados se analizan, se discriminan y se priorizan para caracterizar el uso de la energía partiendo de la realidad nacional y definiendo la realidad particular del cantón. Muchos de los datos se proyectan para establecer a futuro cuál será la condición energética de Cuenca en años posteriores y se analiza para establecer acciones que permitan fortalecer el buen vivir, el cuidado del medioambiente y el uso eficiente de la energía.

Los puntos presentados al final de la investigación aportaran de manera significativa para determinar las líneas de acción en investigación, desarrollo e innovación que emprenderá la Universidad Politécnica Salesiana en el ámbito de la energía a través del CIAME (Centro de Investigación en Automatización, Materiales y Energía).

Palabras clave: energía, cantón Cuenca, eficiencia energética, CIAME

\section{Abstract}

This article is based on a data compilation at the Canton Cuenca Ecuador and in regard to generation and use of electricity and the use of fossil fuels. All collected data are analyzed, and prioritized discriminate to characterize the use of energy based on the national reality and defining the particular reality of the Canton. Many of the data is projected forward to establish what will be the energetic condition of Cuenca in later years and analyzed to establish actions to strengthen the good life, care for the environment and the efficient use of energy.

The points presented the end of the investigation contributed significantly to shaping the action in research, development and innovation undertaken by the Salesian Polytechnic University in the field of energy through the CIAME (Centre for Research in Automation, Materials and Energy).

Keywords: energy, Canton Cuenca, energy effciency, $C I A M$

\footnotetext{
* Máster en Gestión y Auditorias Ambientales, Ingeniero Mecánico, Profesor de la Carrera de Ingeniería Mecánica -UPS- sede Cuenca, estudiante de Doctorado en Ingeniería de Proyectos, Universidad Tecnológica de Panamá.

** Ingeniero Mecánico Automotriz, Profesor de la de la Carrera de Ingeniería Mecánica Atomotriz -UPS- sede Cuenca.

Recibido: 29-julio-2011; Aprobado: 5-octubre-2011
}

Forma sugerida para citar: Calle Sigüencia, John. (2011). "Situación Energética del Cantón Cuenca y líneas de acción preferentes para fortalecer la investigación y el desarrollo a nivel local.". INGENIUS. № 6, (julio/diciembre). pp. 85-95 .ISSN: 1390-650X 


\section{Introducción}

Cuenca, ubicada al sur de Ecuador, es uno de los quince cantones que conforman la provincia del Azuay. A una cota media de 2550 msnm, se asienta en la hoya del río Paute, entre las coordenadas $78^{\circ} 59^{\prime}$ - $79^{\circ} 01^{\prime}$ de longitud oeste y $2^{\circ} 52^{\prime}-2^{\circ} 54^{\prime}$ de latitud sur, hacia el centro-sur de la cordillera de los Andes.

El cantón Cuenca se ha constituido en uno de los principales ejes del desarrollo económico del Ecuador. Su producto interno bruto (PIB) per cápita a diciembre de 2010 se estimó en US\$ 7951,87, en tanto su población alcanzó los 505585 habitantes.

La energía utilizada para el desarrollo del sector tanto doméstico, comercial e industrial proviene principalmente de fuentes convencionales en su mayor parte de la generación hidroeléctrica acompañado de generación mediante combustibles fósiles derivados del petróleo como son las gasolinas, el diesel, el búnker y el GLP. El crecimiento demográfico constante y la mayor demanda de energía han provocado que se obligue a un crecimiento en la generación cubriéndose principalmente esta demanda con sistemas hidroeléctricos que son parte de las políticas gubernamentales actuales y que han tenido un desarrollo firme en los últimos años, además se ha fortalecido el uso de derivados del petróleo especialmente las gasolinas en lo que respecta a medios de transporte y el GLP en lo que concierne al consumo doméstico e industrial especialmente en la producción de agua caliente sanitaria (ACS) para cubrir necesidades básicas de aseo y procesos industriales para transformación de materias primas.

El uso eficiente de la energía está condicionado por algunos factores que se los debe ges- tionar de manera adecuada para fortalecer las políticas del buen vivir y conseguir solventar las necesidades energéticas sin causar alteraciones graves al medioambiente, en este contexto la investigación, el desarrollo y la innovación $(\mathrm{I}+\mathrm{D}+\mathrm{i})$ se convierten en pilares fundamentales en el logro de este objetivo y contribuyen sin duda alguna al avance de la sociedad en la búsqueda de la sostenibilidad y conservación del planeta

El análisis realizado tiene como base los sistemas energéticos utilizados en el cantón Cuenca partiendo de la matriz nacional energética del Ecuador con la información de ministerios y organismos gubernamentales que permiten cuantificar el tipo de consumo en lo que respecta a producción y uso final que se le da a la energía en los diferentes ámbitos del desarrollo social para de esta manera proyectar líneas de acción que permitan a Universidades, ministerios del ramo, centros de investigación estatales y particulares promover proyectos que favorezcan el uso adecuado y eficiente de la energía en la búsqueda de alternativas viables que aporten al desarrollo de la región.

\section{Materiales y métodos}

Para caracterizar la situación energética en el cantón Cuenca se realiza un análisis de la situación actual en lo referente a la generación y uso de la energía eléctrica y también la utilización de combustibles fósiles ya sea para generación eléctrica u otras aplicaciones industriales especialmente en la producción de fluidos caloportadores que se utilizan en mecanismos de intercambio de calor en procesos domésticos e industriales; los datos obtenidos son parte de estudios, informes, estadísticas que se han obtenido de ministerios, organismos gubernamentales, empresas públicas y que identifican

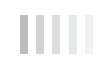


la realidad en lo que se refiere a la situación energética de la localidad en estudio.

En primer lugar se han considerado los datos nacionales de generación eléctrica, en la figura
1 se presente la situación de generación y su proyección hasta el año 2011.
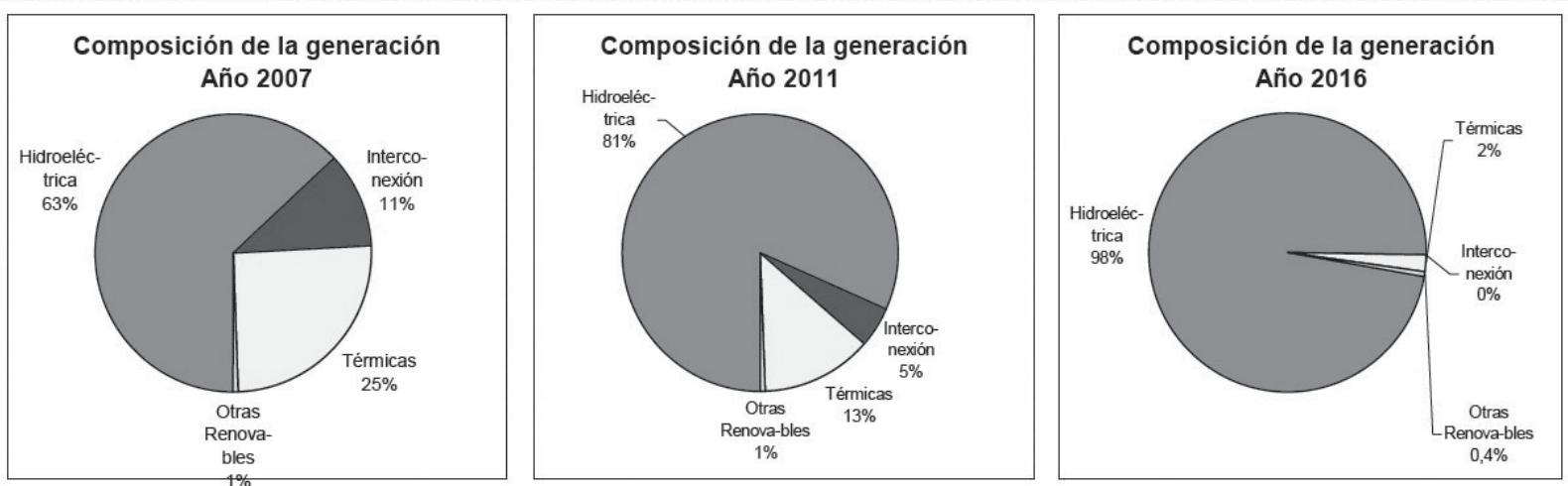

Fig. 1. Composición de generación eléctrica en el Ecuador

Fuente: Presentación realizado por PhD. Miguel Calahorrano Camino, Director de planificación del CONELEC, mayo del 2007 en el Seminario de Energías Renovables organizado por la CGA de la Municipalidad de Cuenca.

Otro de los aspectos que se consideran es el balance de energía y principales características de las empresas eléctricas distribuidoras en el año 2006 tomado del informe estadístico del sector eléctrico ecuatoriano emitido por el CONELEC, periodo 1990-2006, el mismo se muestra en la figura 2.
Otro de los elementos fundamentales a considerar es que el estado ecuatoriano para garantizar el suministro de energía se ha fijado metas en la generación hidroeléctrica, la utilización de gas natural y la eliminación de las centrales térmicas como se puede observar en la figura 3 . 


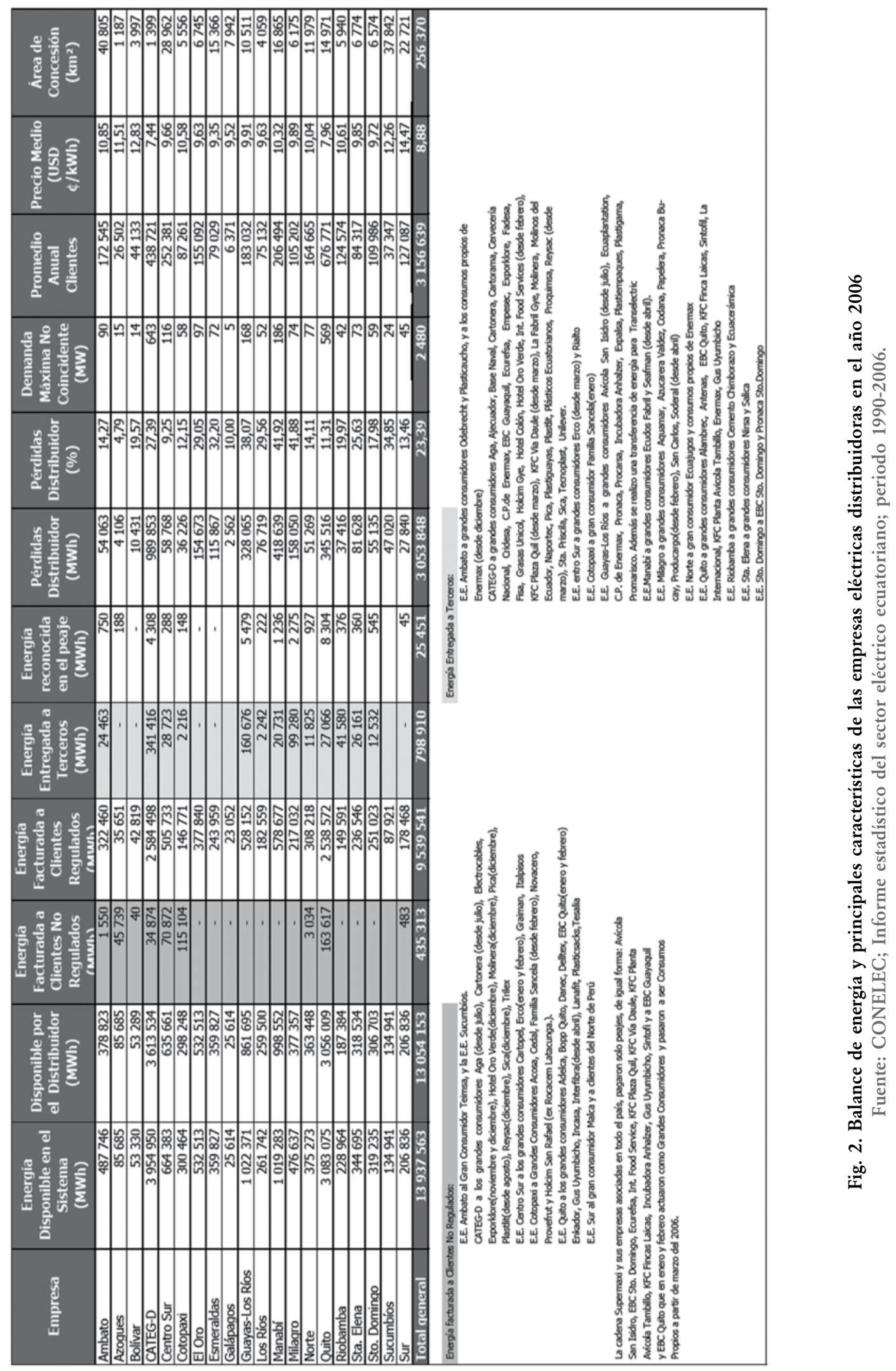




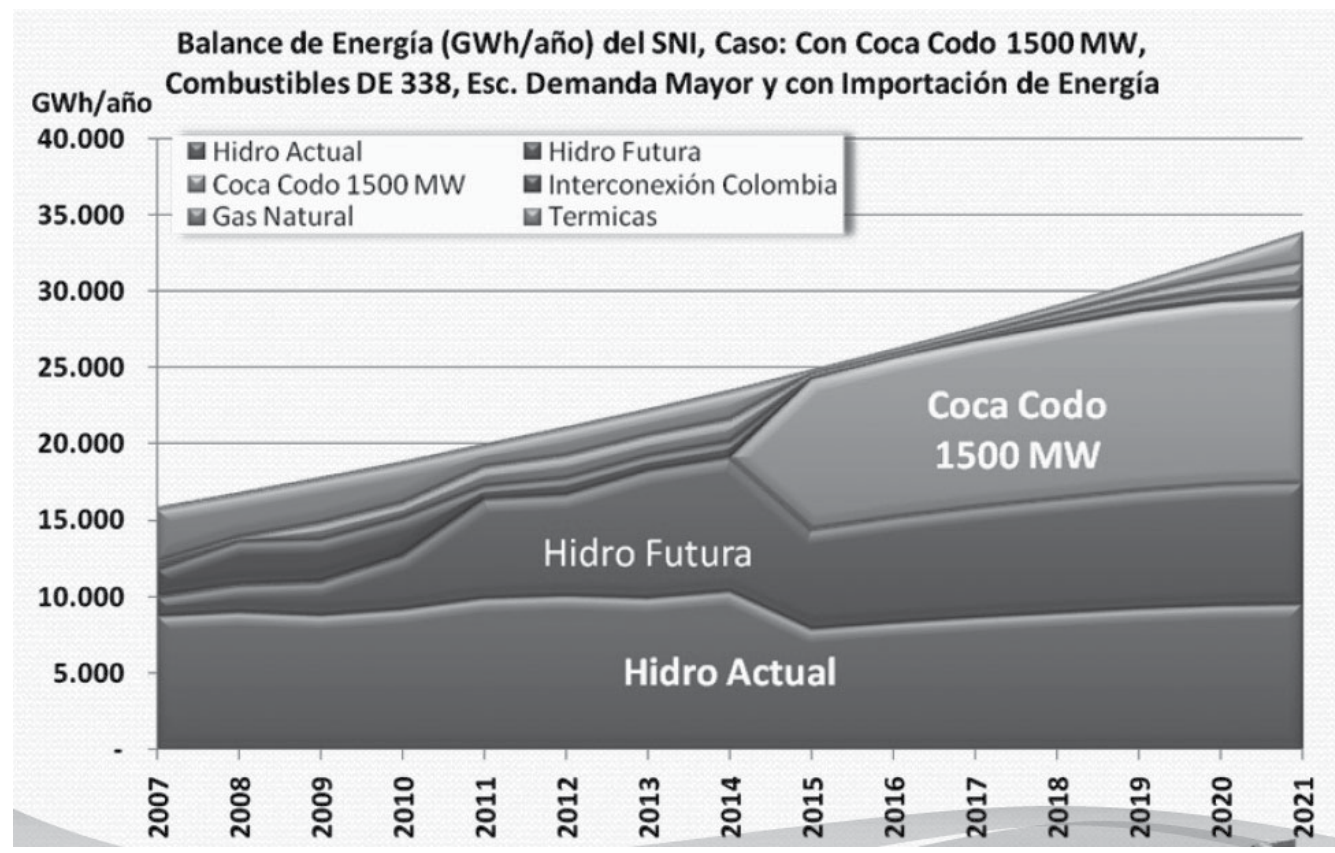

Fig. 3. Evolución de la producción de energía eléctrica hasta el año 2011

Fuente: Presentación realizada por PhD. Miguel Calahorrano Camino, Director de planificación del CONELEC, mayo del 2007, en el Seminario de Energías Renovables organizado por la CGA de la Municipalidad de Cuenca.

En segundo lugar se identifica la situación del cantón Cuenca en lo referente al uso de la energía eléctrica; para esto se ha utilizado los datos de facturación proporcionados por la Empresa Eléctrica Regional Centro Sur (EERCS) del mes de junio del 2011 en donde se puede evidenciar en primera instancia (figura 4) la totalidad de consumo en los sectores domésticos, comercial y empresarial y en la tabla 1 las empresas que utilizan el mayor porcentaje de la energía consumida en este sector.

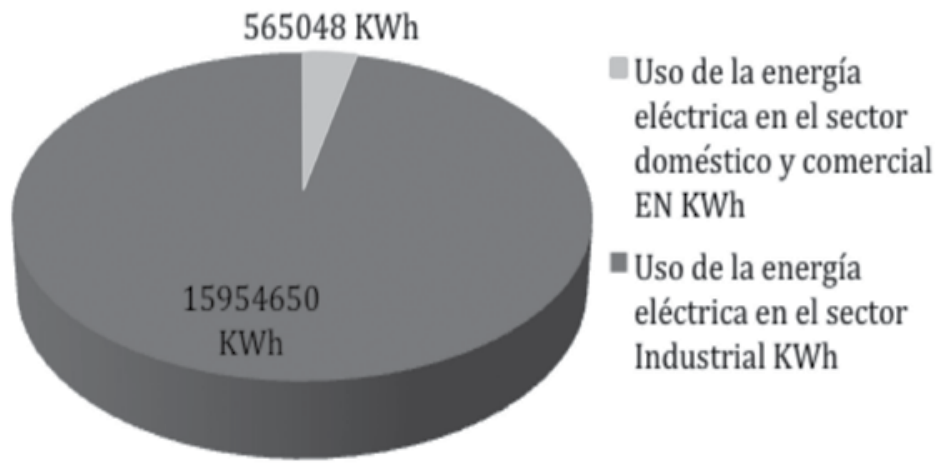

Fig. 4. Consumo de energía eléctrica en sector domestico e industrial del Cantón Cuenca Fuente: Elaboración propia a partir de datos proporcionados por la EERCS en el mes de julio 2011. 
Tabla 1. Consumo de energía eléctrica en el sector industrial del cantón Cuenca

\begin{tabular}{|l|r|r|}
\hline Descripción & Consumo & $\begin{array}{c}\% \\
\text { Consumo }\end{array}$ \\
\hline $\begin{array}{l}\text { CARTONES NACIONALES } \\
\text { S.A.I.CARTOPEL (3897659) }\end{array}$ & 3.282 .240 & $20,57 \%$ \\
\hline GRAIMAN CIA. LTDA. (3898335) & 3.107 .422 & $19,48 \%$ \\
\hline CERÁMICA RIALTO S.A. (3935822) & 988.910 & $6,20 \%$ \\
\hline $\begin{array}{l}\text { ETAPA EP GAPASA-UCUBAMBA } \\
\text { (1877877) }\end{array}$ & 1.057 .950 & $6,63 \%$ \\
\hline ITALPISOS S.A. (3957099) & 785.692 & $4,93 \%$ \\
\hline PLÁSTICOS RIVAL (1395367) & 750.300 & $4,70 \%$ \\
\hline INDUGLOB S.A. (389221) & 507.980 & $3,18 \%$ \\
\hline INSOMET CIA. LTDA. (389403) & 481.774 & $3,02 \%$ \\
\hline PASAMANERÍA S.A. (193508) & 352.513 & $2,21 \%$ \\
\hline ADHEPLAST S.A. (3065240) & 337.022 & $2,11 \%$ \\
\hline LÁCTEOS SAN ANTONIO C.A. (387324) & 282.367 & $1,77 \%$ \\
\hline CERÁMICA ANDINA C.A. (389452) & 284.980 & $1,79 \%$ \\
\hline CERÁMICA RIALTO S.A (389122) & 259.794 & $1,63 \%$ \\
\hline INDUGLOB S.A. (389130) & 219.900 & $1,38 \%$ \\
\hline INSOMET CIA. LTDA. (386078) & 207.245 & $1,30 \%$ \\
\hline ESFEL S.A. (388686) & 127.260 & $0,80 \%$ \\
\hline SINTECUERO S.A. (1121490) & 157.339 & $0,99 \%$ \\
\hline ETAPA EP GAPASA-PLANTA DEL & & \\
CEBOLLAR (290999) & 91.829 & $0,58 \%$ \\
\hline INSOMET CIA. LTDA. (387258) & 126.694 & $0,79 \%$ \\
\hline FCA. LA MURALLA (1194315) & 114.234 & $0,72 \%$ \\
\hline PLASTIAZUAY S.A. (388660) & 107.621 & $0,68 \%$ \\
\hline $\begin{array}{l}\text { INMOBILIARIA PIEDRA HUASI S.A. } \\
\text { (4228896) }\end{array}$ & 98.867 & $0,62 \%$ \\
\hline INSOMET CIA. LTDA. (2042539) & 105.501 & $0,66 \%$ \\
\hline FÁBRICA LA EUROPEA (1395359) & 99.467 & $0,62 \%$ \\
\hline FIBROACERO S.A. (387928) & 65.824 & $0,41 \%$ \\
\hline EMBUANDES CIA.LTDA. (3669223) & 97.448 & $0,61 \%$ \\
\hline EDITORIAL DON BOSCO (175885) & 1.810 .668 & $0,29 \%$ \\
\hline OTROS & 15.954 .650 & $103 \%$ \\
\hline & & \\
\hline
\end{tabular}

Fuente: Elaboración propia a partir de datos proporcionados por la EERCS en el mes de julio 2011.

Para identificar la realidad de la utilización de combustibles en primera instancia se establece la demanda de derivados de petróleo a nivel nacional y su proyección hasta el año 2025 en función de los datos proporcionados por el Ministerio de Minas y Petróleos el mismo que se muestra en la fig. 6 . 


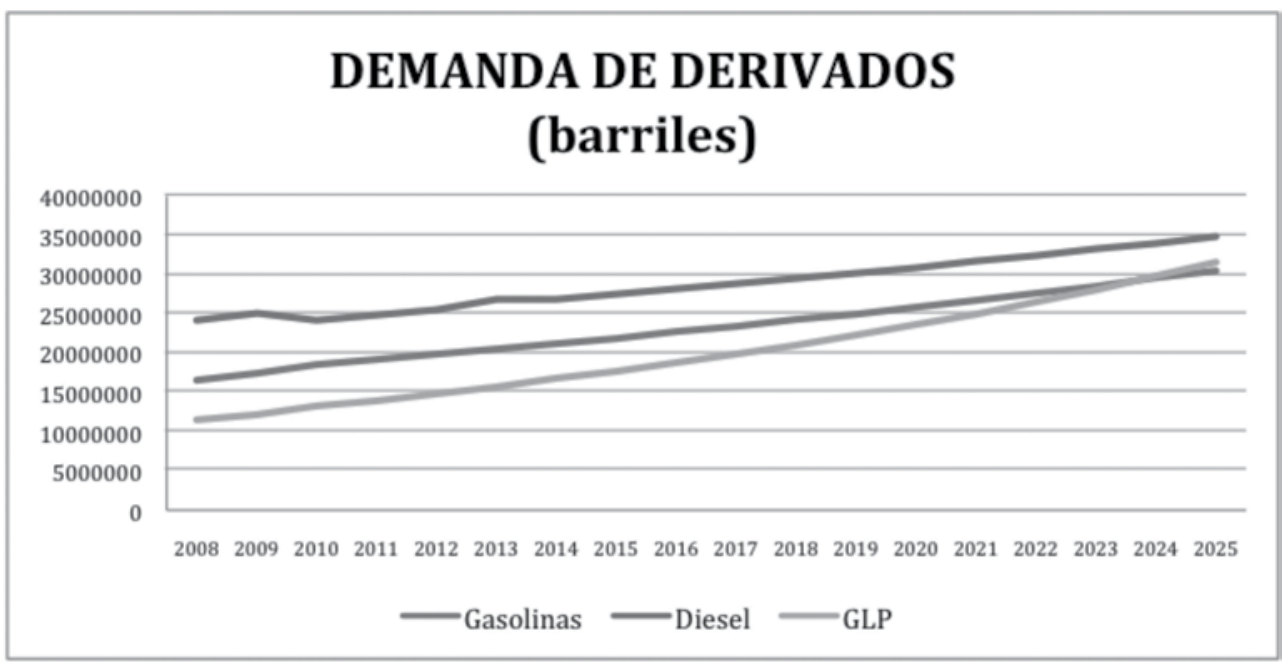

Fig. 6. Proyección de demanda de derivados del petróleo en el Ecuador

Fuente: Elaboración propia en función de datos proporcionados por el Ministerio de Minas y Petróleos del Ecuador.

Además es relevante la información proporcionada por el mismo ministerio la cerca de la calidad de los combustibles en el Ecuador con respecto al \% de azufre, representada en la figura 7.

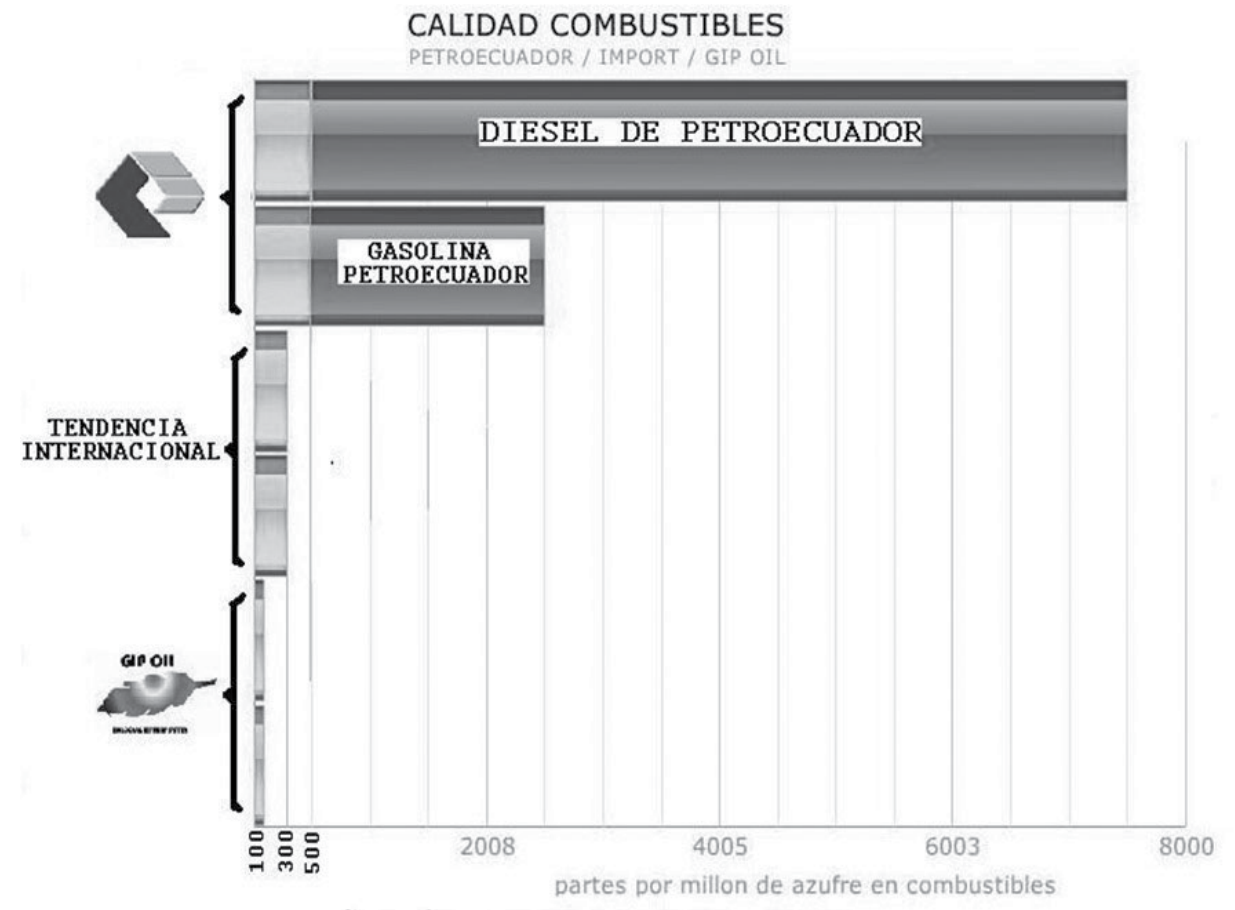

Fig. 7. Calidad de combustibles en el Ecuador

Fuente: Elaboración propia en función de datos proporcionados por el Ministerio de Minas y Petróleos del Ecuador. 
En lo que se refiere a la utilización de combustibles se puede identificar en el estudio realizado por Fundación Natura para el inventario de emisiones del cantón Cuenca elaborado para Cuenca Aire y la Comisión de gestión ambiental del Municipio de Cuenca en el año 2009 los diferentes ámbitos de acuerdo al tipo de combustible. El resumen se muestra en la tabla 2.

Tabla 2. Consumo por uso y tipo de los combustibles en el cantón Cuenca, año 2007

\begin{tabular}{|l|r|r|r|r|r|r|}
\hline & \multicolumn{1}{|c|}{$\begin{array}{c}\text { Gasolina } \\
\text { extra }\end{array}$} & $\begin{array}{c}\text { Gasolina } \\
\text { súper }\end{array}$ & Diesel & Búnker & GLP & Biomasa \\
\hline USO & \multicolumn{2}{|c|}{ galones } & $\mathrm{kg}$ & $\mathrm{m}^{3}$ \\
\hline Tráfico vehicular & 30.827 .979 & 6.200 .929 & 29.034 .561 & & & \\
\hline Industria & & & 3.493 .338 & 3.698 .261 & 16.475 .847 & 1.006 \\
\hline Centrales térmicas & & & 621.442 & 3.852 .730 & & \\
\hline Doméstico & & & & & 48.444 .752 & \\
\hline Fábricas de ladrillos & & & & & & 22.737 \\
\hline TOTAL & $\mathbf{3 0 . 8 2 7 . 9 7 9}$ & $\mathbf{6 . 2 0 0 . 9 2 9}$ & $\mathbf{3 3 . 1 4 9 . 3 4 1}$ & $\mathbf{7 . 5 5 0 . 9 9 1}$ & $\mathbf{6 4 . 9 2 0 . 5 9 9}$ & $\mathbf{2 3 . 7 4 3}$ \\
\hline
\end{tabular}

Fuente: Estudio realizado por Fundación Natura para el inventario de emisiones del Cantón Cuenca elaborado para Cuenca Aire y la Comisión de gestión ambiental del Municipio de Cuenca, 2009.

\begin{tabular}{|l|r|r|r|r|r|}
\hline & $\mathrm{NO}_{\mathrm{x}}$ & $\mathrm{CO}$ & $\mathrm{COV}$ & $\mathrm{SO}_{2}$ & $\mathrm{PM}_{10}$ \\
\hline Tráfico vehicular & $6.214,30$ & $40.377,00$ & $5.437,50$ & 637,2 & 331,2 \\
\hline Vegetación & 0 & 0 & $1.593,70$ & 0 & 0 \\
\hline Industrias & 285,7 & 48,1 & 244,1 & 996 & 79,2 \\
\hline Térmicas & $1.225,80$ & 263,8 & 100,1 & 480,2 & 78,2 \\
\hline Solventes & 0 & 0 & $1.975,40$ & 0 & 0 \\
\hline Gasolineras & 0 & 0 & 925,1 & 0 & 0 \\
\hline GLP doméstico & 157,6 & 24,6 & 5,3 & 0 & 10,4 \\
\hline Canteras & 0 & 0 & 0 & 0 & 39,5 \\
\hline Erosión eólica & 0 & 0 & 0 & 0 & 126,8 \\
\hline Tráfico aéreo & 32,7 & 46,6 & 16,7 & 7,9 & 0,5 \\
\hline Rellenos sanitarios & 0 & 0 & 20,5 & 0 & 0 \\
\hline Ladrilleras & 7,9 & 172,6 & 185,7 & 1,4 & 122,9 \\
\hline
\end{tabular}

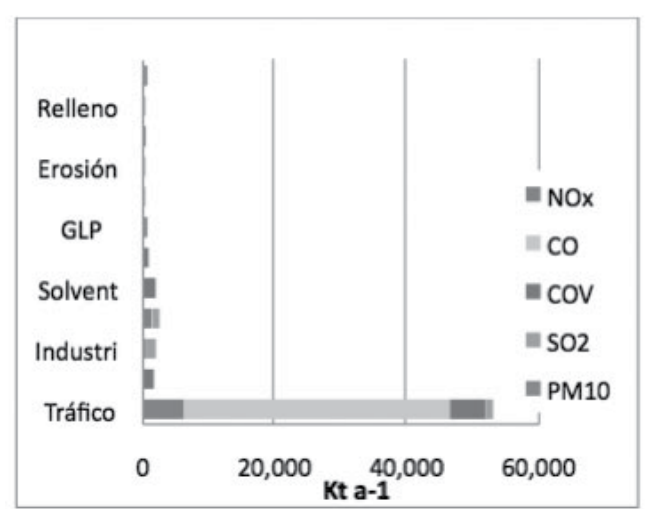

Fig. 8. Emisión de contaminantes primarios del aire por las principales actividades del cantón Cuenca, año 2007

Fuente: Estudio realizado por Fundación Natura para el inventario de emisiones del cantón Cuenca elaborado para Cuenca Aire y la Comisión de Gestión ambiental del Municipio de Cuenca, 2009. 


\section{Resultados y discusión}

Luego de realizar un análisis detallado de los datos presentados se pueden establecer los siguientes criterios:

- La generación hidroeléctrica es una de las fuentes energéticas de desarrollo prioritario en el Ecuador por lo que la mayor cantidad de la demanda de energía eléctrica será cubierta por este sistema.

- La generación a partir de combustibles fósiles en centrales térmicas en su mayor parte desaparecerán o serán reemplazadas por el uso de gas natural que es un combustible más limpio.

- El mayor porcentaje de energía eléctrica en el cantón Cuenca es utilizada en el sector industrial por empresas que transforman materias primas especialmente cartón, papel, cerámicos y plásticos.

- No existe generación a partir de energías no convencionales como la solar, eólica, geotérmica, biomasa u otras.

- Existe una creciente demanda de los derivados de petróleo a nivel nacional especialmente para cubrir la necesidad del sector del transporte, la generación en plantas térmicas, el uso industrial en la producción de calor para transformación de materias primas.

- El GLP es uno de los combustibles de mayor demanda ya sea en ámbito doméstico para la producción de agua caliente sanitaria o en el sector industrial para obtener fluidos caloportadores que se utilizan en intercanbiadores de calor que aportan energía para la transformación de la materia prima.

- El GLP es uno de los combustibles que mayor contaminación provoca por las emanaciones de gases contaminantes durante su combustión.
- El consumo de diésel y gasolinas en el transporte es uno de los contaminantes atmosféricos principales en el Cantón Cuenca debido al contenido de azufre en su estructura.

A partir del análisis realizado se puede evidenciar algunas líneas de acción que se deben emprender para garantizar un uso eficiente de la energía, disminuir la contaminación, garantizar sostenibilidad en el entorno y favorecer el buen vivir de todos los habitantes de la región:

- Fortalecer el desarrollo tecnológico en lo que respecta a generación hidroeléctrica para obtener el mayor grado de eficiencia en la transformación.

- Consolidar la tecnología con el uso de gas natural en la generación de energía eléctrica garantizando eficiencia en la producción y disminuyendo agentes contaminantes producidos con otro tipo de combustibles.

- Desarrollar innovaciones en sistemas energéticos a partir de energías no convencionales como la solar, eólica y biomasa que disminuyan el consumo de energía eléctrica en la obtención de calor y que garanticen eficiencia, costos competitivos, y sostenibilidad en el tiempo

- Desarrollar sistemas innovadores de alta eficiencia; para sistemas que utilizan combustibles fósiles buscando disminuir el consumo de los mismos y la contaminación generada durante su combustión

- Propiciar el desarrollo de combustibles alternativos y de innovaciones tecnológicas que garanticen eficiencia en la transportación y generación mínima de contaminantes considerando un alto rendimiento y durabilidad de los componentes del motor. 
- Propiciar el desarrollo y utilización de sistemas híbridos eficientes (con combustible fósil y energía renovable) para la obtención de ACS para uso doméstico.

- Propiciar el desarrollo la innovación y el uso de sistemas híbridos eficientes (con combustible fósil y energía renovable) para la obtención de fluidos caloportadores que se utilizan en el ámbito industrial.

- Favorecer el desarrollo de tecnología para el uso de biomasa residual que se obtiene de los desperdicios de la utilización de la madera. Al ser Cuenca el mayor productor de muebles y enseres de madera del país, los residuos generados de esta actividad pueden convertirse en una alternativa rentable, eficiente y limpia pues los empresarios de la madera disminuirían o dejarían de utilizar combustibles fósiles para sus procesos.

\section{Referencias bibliográficas}

[1] Calahorrano Camino Miguel PhD. Director de planificación del CONELEC. (2007) "Seminario de Energías Renovables". Comisión de Gestión Ambiental CGA , Municipalidad de Cuenca.

[2] Comisión de Gestión Ambiental, I. Municipalidad de Cuenca (2007). "Plan Energético de Cuenca 2007-2017".

[3] CONELEC. "Informe estadístico del Sector Eléctrico Ecuatoriano” (1990-2006).
[4] CONELEC. Consejo Nacional de Electrificación del Ecuador. (consultado el 5 de septiembre del 2010). Disponible en: http://www.conelec.gob.ec/ contenido.php?cd $=2338 \& \mathrm{l}=1$

[5] EERCS. Empresa Eléctrica Regional Centro Sur (Consultado el 22 de julio del 2011). Disponible en: http://www.centrosur.com.ec/ estad $\%$ C $3 \%$ ADsticas/el-mercado-regulado/ energ\%C3\%AD-consumida

[6] Fundación Natura. (2009) "Inventario de emisiones del Cantón Cuenca" elaborado para Cuenca Aire y la Comisión de gestión ambiental del $\mathrm{Mu}$ nicipio de Cuenca.

[7] Fundación Natura. (2009-2013) Plan de Manejo de Calidad del Aire de Cuenca elaborado por para la ilustre municipalidad del Cantón Cuenca.

[8] Ministerio de Minas y Petróleos. (2009) "Primer foro de Biocombustibles". Cuenca.

[9] NEC Instituto Ecuatoriano de Estadísticas y Censos. http://www.inec.gov.ec/estadistica

[10] Petrocomercial. (2002-2006). Consumo histórico de combustibles en el Cantón Cuenca. 